\title{
Worst-Case Routing Performance Metrics for Sensor Networks
}

\author{
Edwin Soedarmadji \\ California Institute of Technology \\ Pasadena, CA 91125, USA \\ edwin@systems.caltech.edu
}

\begin{abstract}
Successful integration of pervasive sensor networks in mission critical applications depends on the ability of these networks to cope with and reasonably perform under the worst-case scenarios. One of the key performance measures is the network's ability to route information from the source node to the intended destination. This paper introduces a general framework with which worst-case routing performance of sensor networks can be evaluated and compared. Ultimately, our method can either be used as a design optimization tool, or a decision making tool to select and price contending sensor network designs.
\end{abstract}

\section{Introduction}

Originally designed for military applications, sensor networks have steadily entered many areas of civilian applications. While many early civilian sensor networks were limited to non-critical remote environmental monitoring systems, state of the art sensor networks are integrated into many current and future designs of mission critical civilian data monitoring systems.

For example, the Advanced National Seismic System (ANSS) [1] and the Pacific Tsunami Warning System operated by the National Weather Service [2] are two large networks of sensors designed to reliably detect, measure, report, and track large scale, catastrophic geological events moving at the speed of several kilometers per second.

While these systems are wired sensor networks, many future mission critical systems would be pervasive wireless sensor networks designed to detect the presence of dangerous levels of biohazards, radiation, lethal mineshaft gases, etc. In these applications, losing even one symbol, packet, or file can have very disastrous consequences. In most cases, the source node must strive to maximize the probability of successfully transmitting the information to the destination node. Retransmission might be impossible, as the source nodes could have been destroyed or incapacitated.
Motivated by this problem, we introduce a worst-case routing performance metric to measure and compare network path lengths that depend on the paths' probability of failure under the worst-case scenario. Ideally, given a source-destination pair, the path that minimizes this length is selected for data transmission. The minimum path length over all source-destination pairs can then be used to evaluate the network's worst case routing performance. For brevity, we will refer to this metric as the Worst-Case Error (WCE) metric, where "Error" can represent any types of failure.

In any metric, a path's length depends on its edges' lengths, and our WCE metric is no exception. However, the WCE length also depends on how each network link is modeled and parametrized. In this paper, we provide three network models where each link is modeled as a $q$ ary Symmetric Channel (q-SC), a $q$-ary Erasure Channel, and a non-negative mean AWGN channel. These channels are parametrized by their symbol error ratio, symbol erasure ratio, and the mean-variance pair. For convenience, we consider these parameters as edge metrics referred to as the "Bit Error Ratio" (BER) - which is quite a misnomer because our model is not restricted to binary channels, and the parameter is not always a ratio.

To the best of our knowledge, we believe that our approach of using the BER and WCE metric to evaluate the worst-case routing performance of pervasive sensor networks is new. Our algorithm is applicable to many other network optimization problems: although here we use the WCE metric for evaluating sensor networks, it can be used in other network problems that attempt to minimize the worst-case occurence probability of non-typical, but highly catastrophic data transmission failures.

\section{Formulation and Notation}

The network is modeled with a digraph $G=(V, E)$, where $V, E$, and $\Pi$ are the node, edge and path sets of $G$. Two nodes $s$ and $d \in V$ are the source and destination nodes, and $\Pi \subset \Pi$ denotes the set of all paths from $s$ to $d$. 
A path $\pi \in \Pi$ whose nodes $V_{\pi} \subset V$ are connected by $E_{\pi} \subset E$ is denoted by either $\left\langle v_{0}, \ldots, v_{J}\right\rangle,\left\langle e_{1}, \ldots, e_{J}\right\rangle$, or $\left\langle v_{0}, e_{1}, \ldots, e_{J}, v_{J}\right\rangle$. The number of nodes (or edges) in $\pi$ is denoted by $|\pi|_{v}$ (or $|\pi|_{e}$ ). The symbol $\left\langle v_{i}, v_{i+1}\right\rangle$ denotes the edge (path) connecting the two (non-) adjacent nodes $v_{i}$ and $v_{i+1}$. A partial path $\pi_{j}$ of $\pi$ denotes $\left\langle v_{0}, \ldots, v_{j}\right\rangle$, with $0<$ $j \leq J$, and a truncated path $\bar{\pi}_{j}$ is $\left\langle v_{0}, e_{1}, \ldots, v_{j-1}, e_{j}\right\rangle$.

Denote the message by $B \in \mathcal{B}$, where $\mathcal{B}$ is the space of all allowable messages in the network. Let $B_{i}$ denote the value of $B$ as it departs from $v_{i}$; and let $\bar{B}_{i}$ denote the value of $B$ as it leaves $e_{i}$. Both $v_{i}$ and $e_{i}$ are parts of $\left\langle v_{0}, e_{1}, \ldots, e_{J}, v_{J}\right\rangle$, along which $B$ evolves as follows:

$$
B_{0} \stackrel{e_{1}}{\longrightarrow} \bar{B}_{1} \stackrel{v_{1}}{\longrightarrow} B_{1} \stackrel{e_{2}}{\longrightarrow} \bar{B}_{2} \stackrel{v_{2}}{\longrightarrow} \cdots \stackrel{e_{J}}{\longrightarrow} \bar{B}_{J} \stackrel{v_{J}}{\longrightarrow} B_{J}
$$

where $v_{i}$ and $e_{i}$ correspond to the operators $\boldsymbol{v}_{i}, \boldsymbol{e}_{i} \in \mathcal{E}$ : $\mathcal{B} \rightarrow \mathcal{B}$ given by $B_{i}=\boldsymbol{v}_{i}\left(\bar{B}_{i}\right)$ and $\bar{B}_{i+1}=\boldsymbol{e}_{i}\left(B_{i}\right)$. The operator for $\pi$ is $\boldsymbol{\pi}=\boldsymbol{v}_{J} \circ \boldsymbol{e}_{J} \circ \cdots \circ \boldsymbol{e}_{1} \circ \boldsymbol{v}_{0}$. For $\pi_{j}$, it is $\boldsymbol{\pi}_{j}=\boldsymbol{v}_{j} \circ \boldsymbol{e}_{j} \circ \cdots \circ \boldsymbol{e}_{1} \circ \boldsymbol{v}_{0}$, and for $\bar{\pi}_{j}$, it is $\overline{\boldsymbol{\pi}}_{j}=\boldsymbol{e}_{j} \circ$ $\boldsymbol{v}_{j-1} \cdots \circ \boldsymbol{e}_{1} \circ \boldsymbol{v}_{0}$. Thus, $\boldsymbol{\pi}_{j}\left(B_{0}\right)=B_{j}$ and $\overline{\boldsymbol{\pi}}_{j}\left(B_{0}\right)=\bar{B}_{j}$.

Define the function $X: \mathcal{B} \times \mathcal{B} \rightarrow \mathcal{M}$ that measures the distance $x \in \mathcal{M}$ between a message $B_{0}$ at $v_{0}$ which evolves into $B_{J}$ at $v_{J}$, where $v_{0}$ and $v_{J}$ are connected by $\left\langle v_{0}, v_{J}\right\rangle$, and $\mathcal{M}$ is the metric space. The distance $x$ from $B_{i}$ to $B_{i^{\prime}}$ is denoted by $x=X_{i, i^{\prime}}=X\left(B_{i}, B_{i^{\prime}}\right)$, and $X_{i, \bar{i}^{\prime}}$ is a shorthand for $X\left(B_{i}, \bar{B}_{i^{\prime}}\right)$. If $e=\left\langle v_{i}, v_{i^{\prime}}\right\rangle$ then we define the shorthand $X(e)=X\left(B_{i}, \bar{B}_{i^{\prime}}\right)$.

Often, apart from $B_{0}$, the messages $B_{i}$ at $v_{i}$ are random variables. Consequently, $X_{0, i}$ are also random variables. Consider the random variable $x=X_{i, i^{\prime}}$ that measures the distance between the message at $v_{i}$ and its image at $v_{i^{\prime}}$. Define $P(x, \boldsymbol{\lambda})$ as the probability density of $x$ parameterized by a vector $\boldsymbol{\lambda} \in \Lambda$. For example, for $x \in \mathbb{R}$ and $P$ Gaussian, $\boldsymbol{\lambda}$ is the vector of $P$ 's mean and variance $\left(\mu, \sigma^{2}\right)$. The value $\boldsymbol{\lambda}=\infty$ is reserved to denote the absence of connection between two nodes. Each edge $e_{i}$ in the network can be characterized by its parameter $\boldsymbol{\lambda}_{i}$

Finally, we define the worst case function $\bar{x}\left(\boldsymbol{\lambda}_{i}, \epsilon\right)$ that computes, given a probability density $P\left(x, \boldsymbol{\lambda}_{i}\right)$, the worst case "possible" value of $x$, where "possible" values $y$ are defined as those $y$ values with probability $P\left(y, \boldsymbol{\lambda}_{i}\right)>\epsilon$.

$$
\bar{x}\left(\boldsymbol{\lambda}_{i}, \epsilon\right)=\max _{x}\left\{x \mid P\left(x, \boldsymbol{\lambda}_{i}\right) \geq \epsilon, x \in \mathcal{M}\right\}
$$

Consider a path $\pi=\left\langle e_{1}, \ldots, e_{J}\right\rangle \in \Pi$ and its partial path $\pi_{j}=\left\langle e_{1}, \ldots, e_{j}\right\rangle$ with $1 \leq j \leq J$. For convenience, we also define the function $\beta: \Pi \rightarrow \Lambda$ that maps a path $\pi$ (or an edge $e_{i}$ ) into a density parameter $\boldsymbol{\lambda}_{\pi}$ (or $\boldsymbol{\lambda}_{i}$ ) and the function $\omega: \Pi \rightarrow \mathcal{M}$ that maps a path or an edge (given $\epsilon$ ) into its worst case value $x \in \mathcal{M}$. For $e_{i}$, the $\beta$ and $\omega$ are related to $\bar{x}\left(\boldsymbol{\lambda}_{i}, \epsilon\right)$ through: $\omega\left(e_{i}\right)=\bar{x}\left(\beta\left(e_{i}\right), \epsilon\right)$. For $\pi$, assuming $\boldsymbol{\lambda}_{\pi}$ is defined, similarly we have $\omega(\pi)=\bar{x}(\beta(\pi), \epsilon)$. The next question is, how does $\boldsymbol{\lambda}_{\pi}$ depend on $\boldsymbol{\lambda}_{i}$ 's, and how does $\bar{x}\left(\boldsymbol{\lambda}_{\pi}, \epsilon\right)$ depend on $\bar{x}_{i}=\bar{x}\left(\boldsymbol{\lambda}_{i}, \epsilon\right)$ ?
Let us assume that the addition operation is defined in $\Lambda$ and $\mathcal{M}$ and is denoted by $\oplus$. If $x_{1}=X\left(e_{1}\right), x_{2}=X\left(e_{2}\right)$, $\boldsymbol{\lambda}_{1}=\beta\left(e_{1}\right), \boldsymbol{\lambda}_{2}=\beta\left(e_{2}\right), \bar{x}_{1}=\omega\left(e_{1}\right), \bar{x}_{2}=\omega\left(e_{2}\right)$, and $\pi=\left\langle e_{1}, e_{2}\right\rangle$, then we say $x_{\pi}=x_{1} \oplus x_{2}, \boldsymbol{\lambda}_{\pi}=\boldsymbol{\lambda}_{1} \oplus \boldsymbol{\lambda}_{2}$, or $\bar{x}_{\pi}=\bar{x}_{1} \oplus \bar{x}_{2}$. For these expressions to make sense, the $\oplus, \Lambda$ and $\mathcal{M}$ have to obey the properties outlined in the next section. With $\oplus$, we can now define $x_{\pi}, \lambda_{\pi}$ and $\bar{x}_{\pi}$ in terms of $x_{i}, \boldsymbol{\lambda}_{i}$ and $\bar{x}_{i}$ using a generalized summation: $x_{\pi}=\bigoplus x_{i}, \boldsymbol{\lambda}_{\pi}=\bigoplus \boldsymbol{\lambda}_{i}$ and $\bar{x}_{\pi}=\bigoplus \bar{x}_{i}$. The pairings of $\Lambda$ and $\mathcal{M}$ with $\oplus$ form what we call the $\mathbf{X}, \mathbf{B}$, and $\mathbf{W}$ algebras, from the $X, \beta$, and $\omega$ functions, respectively.

Between two nodes, the optimal path $\pi^{*}$ is the path with the "shortest" path length from $s$ to $d$ when measured in the $\mathbf{X}, \mathbf{B}$ or $\mathbf{W}$ algebra (or metric). However, having $\oplus, X, \beta$, and $\omega$ is not enough to calculate $\pi^{*}$. We need to compare path lengths. Therefore we need a total order $\preceq$ on $\Lambda$ and $\mathcal{M}$ to evaluate expressions like $x_{\pi} \preceq x_{\pi^{\prime}}, \boldsymbol{\lambda}_{\pi} \preceq \boldsymbol{\lambda}_{\pi^{\prime}}$, or $\bar{x}_{\pi} \preceq \bar{x}_{\pi^{\prime}}$. Once $\preceq$ is defined, then we have:

$$
\begin{aligned}
x^{*} & =\min _{\pi}\left\{x_{\pi} \mid \pi \in \Pi\right\} \\
\boldsymbol{\lambda}^{*} & =\min _{\pi}\left\{\boldsymbol{\lambda}_{\pi} \mid \pi \in \Pi\right\} \\
\bar{x}^{*} & =\min _{\pi}\left\{\bar{x}_{\pi}=\bar{x}\left(\boldsymbol{\lambda}_{\pi}, \epsilon\right) \mid \pi \in \Pi\right\}
\end{aligned}
$$

Example 1 ( $q$-ary Symmetric Channels) Let $Q$ be the $q$ ary alphabet $\{0, \ldots, q-1\}$. Suppose that the source $s$ produces $n$-symbol messages $B \in Q^{n}$, with $B_{l} \in Q$, and $l=1 \ldots n$. Each network link is modeled as a $q$-ary Symmetric Channel (q-SC) with symbol error rate $p$.

Let $B_{i}$ (and $b_{i}=B_{l i}$ ) denote $B$ (and $B_{l}$ ) as it departs from $v_{i}$; and let $\bar{B}_{i}$ (and $\bar{b}_{i}=\bar{B}_{l i}$ ) denote $B$ (and $B_{l}$ ) as it leaves $e_{i}$. The transition probability defining a q-SC is:

$$
P\left(b_{i+1} \mid b_{i}\right)= \begin{cases}1-p & , b_{i}=b_{i+1} \\ p /(q-1) & , b_{i} \neq b_{i+1} .\end{cases}
$$

The operators $\boldsymbol{v}_{i}, \boldsymbol{e}_{i} \in \mathcal{E}: Q^{n} \rightarrow Q^{n}$ given by $B_{i}=$ $\boldsymbol{v}_{i}\left(\bar{B}_{i}\right)$ and $\bar{B}_{i+1}=\boldsymbol{e}_{i}\left(B_{i}\right)$. The function $X$ measures the number of errors (Hamming distance) in $B_{i}$ compared to $B_{i^{\prime}}$, denoted by $x=X_{i, i^{\prime}}=X\left(B_{i}, B_{i^{\prime}}\right)=\mid\left\{l \mid b_{i} \neq\right.$ $\left.b_{i^{\prime}}\right\} \mid$. In a q-SC, the scalar parameter that plays the role of $\boldsymbol{\lambda}$ is $p$, the link symbol error probability. The probability density function $P(x, \boldsymbol{\lambda})=P(x, p)$ is:

$$
P(x, p)= \begin{cases}\left(\begin{array}{l}
n \\
x
\end{array}\right) p^{x}(1-p)^{n-x} & , p \in(0,1) \\
\delta(x) & , p=0 \\
\delta(x-n) & , p=1, \infty\end{cases}
$$

The $\mathbf{B}$ algebra is such that two adjacent edges $e_{1}$ and $e_{2}$ with parameters $\boldsymbol{\lambda}_{1}$ and $\boldsymbol{\lambda}_{2}$ can be viewed as a single edge with parameter $\boldsymbol{\lambda}=\boldsymbol{\lambda}_{1} \oplus \boldsymbol{\lambda}_{2}=p_{1} \oplus p_{2}$ defined by:

$$
\begin{aligned}
& p_{1} \oplus p_{2}=1-\left(1-p_{1}\right)\left(1-p_{2}\right)-\left(p_{1} p_{2}\right) /(q-1) \\
& \bar{x}_{1} \oplus \bar{x}_{2}=\max \left\{x \mid P\left(x, p_{1} \oplus p_{2}\right) \geq \epsilon\right\}
\end{aligned}
$$


Example 2 ( $\boldsymbol{q}$-ary Erasure Channels) The symbol $q-1$ in $Q$ is designated as a special erasure symbol. Each network link is modeled as a $q$-ary Erasure Channel (q-EC) with symbol erasure rate $p$. $B$ can be defined as a block with $n$ packets of $m$ symbols each, and erasures represent lost packets. The transition probability for the q-EC is:

$$
P\left(b_{i+1} \mid b_{i}\right)= \begin{cases}1-p & , b_{i}=b_{i+1} \\ p & , b_{i} \neq b_{i+1}=q-1 .\end{cases}
$$

The function $X$ measures the number of erasures in $B_{i}$ relative to $B_{i^{\prime}}$, denoted by $x=X_{i, i^{\prime}}=X\left(B_{i}, B_{i^{\prime}}\right)=$ $\left|\left\{l \mid b_{i} \neq b_{i^{\prime}}=q-1\right\}\right|$. The probability density function $P(x, \boldsymbol{\lambda})$ is the same as in Example 1, except $p$ is the link symbol erasure probability and the $\mathbf{B}$ algebra is $\boldsymbol{\lambda}=\boldsymbol{\lambda}_{1} \oplus \boldsymbol{\lambda}_{2}=p_{1} \oplus p_{2}$ defined by:

$$
p_{1} \oplus p_{2}=1-\left(1-p_{1}\right)\left(1-p_{2}\right)
$$

Example 3 (Non-negative-mean AWGN) In this example, the message is a scalar $B \in \mathbb{R}^{+}$with non-negativemean AWGN on each link. This message could represent the amount of degradation a packet has experienced so far. Therefore, the source always transmits $B_{0}=0$.

The AWGN is characterized by $\boldsymbol{\lambda}=\left(\mu \geq 0, \sigma^{2}\right)$, where $\mu$ is the mean, and $\sigma^{2}$ the variance of the Gaussian density and the transition probability density is given by:

$$
\begin{aligned}
P\left(B_{i+1} \mid B_{i}\right) & =P\left(x ; \mu, \sigma^{2}\right) \\
& =\frac{1}{\sqrt{2 \pi \sigma^{2}}} \exp \left(\frac{-(x-\mu)^{2}}{2 \sigma^{2}}\right)
\end{aligned}
$$

where $x=B_{i+1}-B_{i}$. Two independent, adjacent edges $e_{1}$ and $e_{2}$ with parameters $\boldsymbol{\lambda}_{1}$ and $\boldsymbol{\lambda}_{2}$ can be treated as a single edge with parameter $\boldsymbol{\lambda}=\boldsymbol{\lambda}_{1} \oplus \boldsymbol{\lambda}_{2}=\boldsymbol{\lambda}_{1}+\boldsymbol{\lambda}_{2}$, where the + sign is the standard vector summation operator. Since $x$ is now continuous, we can solve for $\bar{x}$ analytically by solving

$$
\bar{x}=P^{-1}\left(\epsilon ; \mu, \sigma^{2}\right)=\mu+\sqrt{-\sigma^{2} \ln \left(2 \pi \epsilon^{2} \sigma^{2}\right)},
$$

which has one solution if $2 \pi \sigma^{2}=\epsilon^{-2}$, two solutions $\bar{x}^{-}$ and $\bar{x}^{+}$if $2 \pi \sigma^{2}<\epsilon^{-2}$, and none if $2 \pi \sigma^{2}>\epsilon^{-2}$. Since $\bar{x}$ represents the worst case value for $x$, we always assume $\bar{x}=\bar{x}^{+}$. From this result, we can also compute:

$$
\bar{x}_{1} \oplus \bar{x}_{2}=P^{-1}\left(\epsilon ; \mu_{1}+\mu_{2}, \sigma_{1}^{2}+\sigma_{2}^{2}\right) .
$$

It is useful to think of $\lambda$ 's as $2 \mathrm{D}$ vectors in a half strip $\Lambda=$ $\mathbb{R} \times\left\{\sigma^{2} \leq \epsilon^{-2} /(2 \pi)\right\}$, and the function $\bar{x}$ as an element in $\overline{\mathcal{X}}_{\epsilon}: \Lambda \rightarrow \mathbb{R}$, defining isocontours for each value of $\bar{x}$.

A $\sigma^{2}=0$, the value $\bar{x}$ reaches its limit of $\mu$. If we denote $G$ 's maximum path variance by $\sigma_{\max }^{2}$, then we must have $\sigma^{2} \leq \sigma_{\max }^{2}=\epsilon_{\max }^{-2} /(2 \pi)$ so that $\forall \boldsymbol{\lambda} \in \Lambda$, the value $\bar{x}$ exists. For now, we claim that $\sigma_{\max }^{2}$ is computed with the longest path algorithm on $G$ with edge metrics $\sigma_{i}^{2}$ and usual addition. Later, $\sigma_{\max }^{2}$ is shown to guarantee closure on $\Lambda$.

\section{Generalized Dijkstra's Algorithm}

The problem of finding the optimal path that minimizes the metric of choice $(x, \boldsymbol{\lambda}, \bar{x}$, or others) in a DTN that is represented by a graph $G$ can be solved using the Generalized Dijkstra's Algorithm (GDA) below [3]:

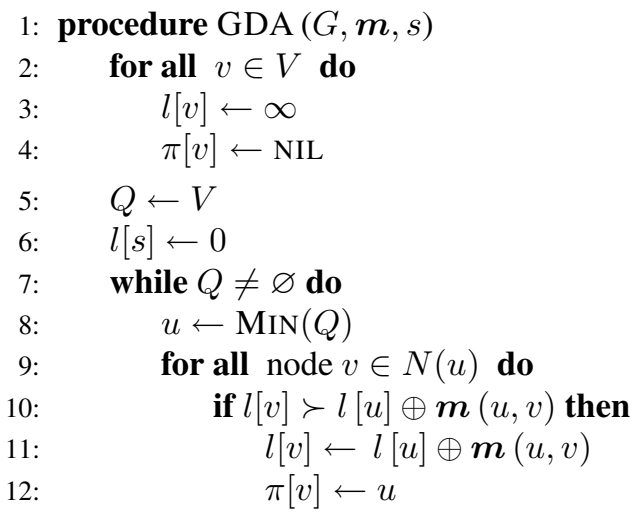

The GDA is practically identical to the Dijkstra's Algorithm (DA) except for the relaxation step, where $\oplus$ and $\preceq$ operators act on a general metric space $\mathcal{M}$ (instead of the equivalent step in DA, where + and $\leq$ operators act on $\mathbb{R}$ ).

On line 9, $N(u)$ denotes the set of all nodes adjacent to $u$. The argument $\boldsymbol{m}$ is the BER lengths of the edges in $G$ each of which is an element in $\mathcal{M}$, and $\boldsymbol{m}(u, v)$ is the BER length of $\langle u, v\rangle$. Lines 10-12 perform the relaxation step of the GDA. This step depends on the definitions of $\mathcal{M}, \oplus$, and $\preceq$. If the GDA (in)correctly returns the path in $G$ with minimum length measured in $\mathcal{M}$, then $(\mathcal{M}, \oplus)$ and $\preceq$ are said to be (in)compatible with the GDA. The following is the required properties for compatibility:

Proposition 1 An algebra $\mathbf{A}=(\mathcal{M}, \oplus)$ and a total order $\preceq$ is compatible with the GDA if and only if it satisfies all the properties in the set denoted by $\mathbf{P}$ below:

$\mathbf{P 1}$ is a commutative monoid, that is, for $a, b, c \in \mathcal{M}$ :

- $\mathcal{M}$ is closed under $\oplus: a \oplus b \in \mathcal{M}$;

- $\oplus$ is associative : $a \oplus(b \oplus c)=(a \oplus b) \oplus c$;

- 0 is the identity : $a \oplus 0=0 \oplus a=a$;

- $\oplus$ is commutative : $a \oplus b=b \oplus a$.

P2 There exists $\infty \in \mathcal{M} \mid a \oplus \infty=\infty \oplus a=\infty$.

$\mathbf{P 3} \preceq$ is a total order on $\mathcal{M}$, i.e., $\preceq$ is :

- reflexive: $a \preceq a$;

- anti-symmetric: if $a \preceq b$ and $b \preceq$ a then $a=b$;

- transitive: if $a \preceq b$ and $b \preceq c$ then $a \preceq c$;

- total: for every $a, b \in \mathcal{M}$ either $a \preceq b$ or $b \preceq a$.

P4 There exists the least element 0 that satisfies $0 \preceq a$.

P5 $a \oplus c \prec b \oplus c$ if $a \prec b$ and $c \in \mathcal{M}-\{\infty\}$.

PROOF: Refer to [3] for a complete proof.

Next, we prove that the $\mathbf{B}$ algebra from examples 1-3 are compatible with the GDA. 
Theorem 2 The algebras $\mathbf{B}=(\Lambda, \oplus)$ defined in examples $1-3$ and their respective total orders $\preceq$ satisfy all the properties in $\mathbf{P}$, and thus compatible with the GDA.

PROOF: The proofs for q-SC and q-EC are omitted because they are mostly algebraic. For AWGN, since $\Lambda \subset$ $\mathbb{R}^{2+}$ and $\mathbb{R}^{2+}$ is not a totally ordered set, we must define $\preceq$ in terms of $\bar{x}(\boldsymbol{\lambda}, \epsilon)$ as follows:

$$
\begin{aligned}
\boldsymbol{\lambda} \preceq \boldsymbol{\lambda}^{\prime} \Leftrightarrow & \bar{x}(\boldsymbol{\lambda}, \epsilon)<\bar{x}\left(\boldsymbol{\lambda}^{\prime}, \epsilon\right) \text { or } \\
& \bar{x}(\boldsymbol{\lambda}, \epsilon)=\bar{x}\left(\boldsymbol{\lambda}^{\prime}, \epsilon\right) \text { and } \sigma^{2} \leq \sigma^{\prime 2}
\end{aligned}
$$

In addition, we also define $\Lambda$ as follows:

$\Lambda=\left(\mathbb{R}^{+} \times\left[0, \sigma_{\text {max }}^{2}\right] \cap\left\{\boldsymbol{\lambda}: \frac{\mu}{\sigma^{2}} \geq\left.\frac{\partial \mu}{\partial \sigma^{2}}\right|_{\sigma_{\text {max }}^{2}}\right\}\right) \cup\{\infty\}$

$\Lambda$ is depicted by the shaded region in figure 1. For a fixed value of $\sigma^{2}$, along any $\bar{x}$ contour, the slope $\mu^{\prime}=\partial \mu / \partial \sigma^{2}$ is equal for all $\mu$ and is maximized at $\sigma_{\max }^{2}$. If $\boldsymbol{\lambda}, \boldsymbol{\lambda}^{\prime}$ have slopes larger than $\mu^{\prime}$, their sum lies in a higher contour.

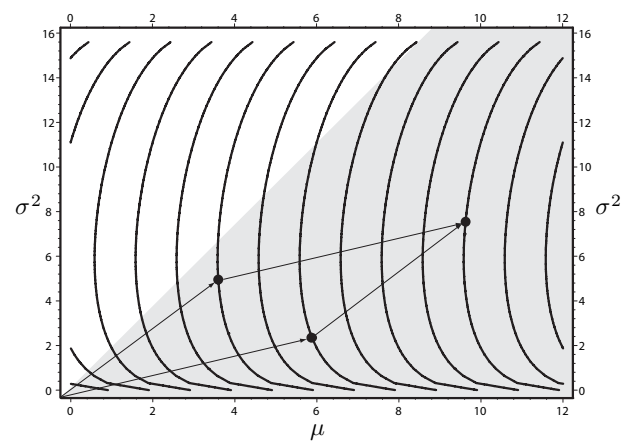

Figure 1: The metric space $\Lambda$ (shaded).

P1 Except for closure, the other monoid properties are obvious because $\oplus$ is the standard vector addition. Because $\Lambda$ does not occupy the full non-negative octant, but rather bounded by $\sigma^{2} \leq \sigma_{\max }^{2}=\epsilon_{\max }^{-2} /(2 \pi)$, in general closure is not guaranteed. However, if we can compute:

$$
\sigma_{\max }^{2}=\max _{\pi \in \Pi}\left\{\sum_{i: v_{i} \in V_{\pi}} \sigma_{i}^{2}\right\}
$$

then closure is guaranteed. This value of $\sigma_{\max }^{2}$ can be obtained by running the DA once on $G$, costing $O\left(V^{2}\right)$. In case one (or both) of the operands is $\infty$, then as in q-SC and q-EC, by definition, the $\oplus$ sum is also $\infty \in \Lambda$.

P2 The proof is derived from closure on $\infty$.

P3 The proof follows from the definition of $\Lambda$ and $\preceq$.

P4 Both terms in equation (9) are minimized when they are zero, i.e., $\mu=0$ and either $\sigma^{2}=0$ or $\sigma^{2}=\epsilon^{-2} /(2 \pi)$. However, only $\boldsymbol{\lambda}=(0,0) \in \Lambda$, and is thus the 0 element. P5 The proof is obvious from figure 1, the definition of $\Lambda$, and concavity of the function $\mu\left(\sigma^{2}\right)$.
Having proven the compatibility of $\mathbf{B}$ to GDA, we now prove that the $\bar{x}$ values in examples 1-3 are non-decreasing functions of their respective $\lambda$ 's, which means that the path with minimum $\boldsymbol{\lambda}$ is also the path with minimum $\bar{x}$.

The situation here is the opposite to the last theorem: the proof for AWGN automatically follows from the definition of $\boldsymbol{\lambda} \preceq \boldsymbol{\lambda}^{\prime}$ and is omitted. In contrast, the proof for q-SC (and q-EC, which is luckily identical) is quite involved:

Lemma 3 For a given $n$ and a fixed $x$, the probability function $P(x, p)$ is maximized at $p=\frac{x}{n}$. Furthermore, $P_{*}=P\left(\left\lfloor\frac{n}{2}\right\rfloor, \frac{1}{2}\right)$ minimizes $P\left(x, \frac{x}{n}\right)$ over all $x \in \mathcal{X}$.

Proof: From the definition of $P(x, p)$ in equation (4), the lemma is true for $x=0$ and $x=n$. Consider :

$$
\frac{\partial P(x, p)}{\partial p}=P(x, p)\left(\frac{x}{p}-\frac{n-x}{1-p}\right)=0
$$

Solving $\left(\frac{x}{p}-\frac{n-x}{1-p}\right)=0$ for $p$ gives us $p=\frac{x}{n}$, which maximizes the function $P(x, p)$ for $x \in(0,1)$.

The next question is, for $0<x<n$, which $x$ minimizes $P\left(x, \frac{x}{n}\right)$ ? Unlike with $p$, we cannot differentiate $P(x, p)$ with respect to $x$ because it is a discrete variable. Instead of approximating $P(x, p)$ with a Gaussian distribution, which is valid only for certain $n$ and $p$, we find the location of the minimum using upper and lower bounds for $\left(\begin{array}{l}n \\ x\end{array}\right)$ in [4] :

$$
\begin{gathered}
\xi(A)=\frac{e^{A} n^{n+\frac{1}{2}}}{(2 \pi)^{\frac{1}{2}}(n-x)^{n-x+\frac{1}{2}} x^{x+\frac{1}{2}}} \\
\lambda_{n x}=\lambda(n, x)=\xi\left(\frac{1}{12 n}-\frac{1}{12 x}-\frac{1}{12(n-x)}\right) \\
\mu_{n x}=\mu(n, x)=\xi\left(\frac{1}{12 n}-\frac{1}{12 x+1}-\frac{1}{12(n-x)+1}\right) \\
\lambda_{n x}<\left(\begin{array}{l}
n \\
x
\end{array}\right)<\mu_{n x}
\end{gathered}
$$

Using (13), $P(x, p)$ is now lower- and upper-bounded by two continuous and differentiable functions of $x$.

$$
\lambda_{n x} p^{x}(1-p)^{n-x}<P(x, p)<\mu_{n x} p^{x}(1-p)^{n-x}
$$

Since $p=\frac{x}{n}$, we substitute $x=n p$ into the equation above and solve the $p$ roots of the $p$ derivatives of both the lower and upper bounds of $P(x, p)$ to find the minima with respect to $p$. The lower and upper bounds are minimized at $p=\frac{1}{2}$. Since $p=\frac{x}{n}$, then $x=\frac{n}{2}$, substituting into the above inequality, we have:

$$
\sqrt{\frac{2}{n \pi}} e^{-\frac{18 n-1}{12 n(6 n+1)}}<P\left(\left\lfloor\frac{n}{2}\right\rfloor, \frac{1}{2}\right)<\sqrt{\frac{2}{n \pi}} e^{-\frac{1}{4 n}}
$$

For large values of $n$ the lower and upper bounds converge. In fact, in (13), $\lambda_{n x}$ converge to $\mu_{n x}$ for all $x$, including at the discrete points $0<x<n \in \mathbb{N}$. Thus the minima for $\lambda_{n x}$ and $\mu_{n x}$ over the continuous $x$ must also be the minimum for $P(x, x / n)$ over the discrete $x$, denoted by $P_{*}=P\left(\left\lfloor\frac{n}{2}\right\rfloor, \frac{1}{2}\right)$

A function $f(x)$ is unimodal over $x \in[a, b]$ if there exists an $x_{0}$ such that $f(x)$ is monotonically increasing for $x<x_{0}$ and monotonically decreasing for $x>x_{0}$. 
Lemma $4 P(x, p)$ is unimodal over $x$ and $p$.

Proof: $\quad$ To prove unimodality over $x$, solve $P(x, p)<$ $P(x+1, p)$ for $x$, giving $x<n p-(1-p)$ or $x<x_{0}=\lfloor n p\rfloor$. Similarly, $P(x, p)>P(x+1, p)$ or $x>x_{0}$. Unimodality over $p$ is proven by differentiating with respect to $p$ :

$$
P^{\prime}(x, p)=\frac{\partial}{\partial p} P(x, p)=P(x, p)\left(\frac{x}{p}-\frac{n-x}{1-p}\right)
$$

$P^{\prime}\left(x, p_{0}\right)=0$ at $p_{0}=\frac{x}{n}$. For $p<p_{0}$, we have $P^{\prime}(x, p)>0$ and for $p>p_{0}$, we have $P^{\prime}(x, p)<0$.

Corrolary 5 The set $\epsilon$ of admissible $\epsilon$ is $\left[0, \bar{\epsilon}=P_{*}\right]$.

Proof: $\quad$ Recall that $\bar{x}(p, \epsilon)=\max \{x \mid P(x, p) \geq \epsilon\}$. To ensure $\bar{x}(p, \epsilon)$ is defined for all $p \in \Lambda$, for each value of $p$ we must have $P(x, p) \geq \epsilon$ for some $x$. This condition is trivially met if $\epsilon \leq 0$ because $P(x, p) \geq 0$. If $\epsilon>P_{*}$, then $\left\{x \mid P\left(x, \frac{1}{2}\right) \geq \epsilon\right\}=\varnothing$, and $\bar{x}\left(p, \frac{1}{2}\right)$ is invalid. Thus, the set of admissible $\epsilon$ is given by $\left[0, P_{*}\right]$.

Denote by $p_{i}(x, \epsilon)=p_{i}(x)=\{p \in \Lambda \mid P(x, p)=\epsilon\}_{i}$ the $i$-th roots of $P(x, p)=\epsilon$ given $n$ and $\epsilon$. From lemma 4, if $\epsilon \in \epsilon$, then there is at least one root. At $x=0$, the single root is $p_{0}(0)=0$ and at $x=n$, it is $p_{1}(n)=1$. Except for another special case when $\epsilon=P_{*}$, where at the midpoint $x_{m}=\left\lfloor\frac{n}{2}\right\rfloor$ there is only one root to $p_{0}\left(x_{m}\right)=p_{1}\left(x_{m}\right)=\frac{1}{2}$, in general, there are two distinct roots $p_{0}(x), p_{1}(x) \in[0,1]$, with $p_{0}(x)<\frac{x}{n}<p_{1}(x)$. If $\epsilon$ goes toward 0 , then $p_{0}(x) \rightarrow$ 0 and $p_{1}(x) \rightarrow 1$, except $p_{1}(0)=0$ and $p_{0}(1)=1$. If $\epsilon$ goes toward $P_{*}$, then $p_{0}(x)$ increases, while $p_{1}(x)$ decreases.

Define the sets $P_{0}$ and $P_{1}$, each having the $n+1$ values of $p_{0}(x)$ and $p_{1}(x)$, for $0 \leq x \leq n$. These values are also referred to as $x_{0}(p)$ and $x_{1}(p)$, for $0 \leq p=\frac{x}{n} \leq n$.

Lemma 6 The roots $p_{0}(x)$ and $p_{1}(x)$ are non-decreasing functions of $x$ with $p_{0}(x)=p_{1}(x)$ only at $x=0$ and $x=n$ (or $x=x_{m}$ for the special case where

Proof: First, observe that $P(x, p)=P(x+1, p)$ only has one root at $p=p^{\times}=\frac{x+1}{n+1}$ between the maxima of $P(x, p)$ and $P(x+1, p)$, i.e., $\frac{x}{n}<p^{\times}<\frac{x+1}{n}$. From lemma 4, this implies that if $p<p^{\times}$then $P(x, p)>P(x+1, p)$, and if $p>p^{\times}, P(x, p)<P(x+1, p)$. Hence, $p_{0}(x) \leq$ $p_{0}(x+1)$ and $p_{1}(x) \leq p_{1}(x+1)$. Therefore, both $p_{0}(x)$ and $p_{1}(x)$ are non-decreasing functions of $x$.

Theorem 7 The values $\bar{x}(p, \epsilon)=\max _{x=n p}\left\{x_{0}(p)\right\}$ and $\max _{x=n p}\left\{x_{1}(p)\right\}$ are non-decreasing functions of $p$.

Proof: First, the $\max _{x}$ function is used because it is possible to have $p_{0}(x)=p_{0}\left(x^{\prime}\right) \in P_{0}$ for $x \neq x^{\prime}$. For example, if $\epsilon=0, p_{0}(0)=\cdots=p_{0}(n-1)=0$ (the same argument applies to $\left.P_{1}\right)$. Just as $\max _{x}$ in $\bar{x}(p, \epsilon)$ isolates the largest $x$ satisfying $P(x, p) \geq \epsilon$, the function $\max _{x} x_{0}(p)$ isolates the largest $x$ satisfying $P(x, p)=\epsilon$. After establishing a unique $x$ for each $p$, the proof follows directly from the monotonicity of $p(x)$.

\section{Conclusion and Discussion}

The preceding results provide us with a practical method to measure the worst-case routing performance of a sensor network. The method is based on the Generalized Dijkstra's Algorithm (GDA), which requires the edge metric to obey a certain set of necessary and sufficient conditions. We provided three examples where the links are modeled as $q$ ary symmetric channels, $q$-ary erasure channels, and nonnegative mean AWGN channels, each with its own edge metric that is nothing more than the parameters of its transmission failure probability density.

By letting the metric to be density parameters, we computed any path length by appropriate combination of its edge lengths according to the laws of probability, thus fully preserving the stochastic nature of the routing problem (instead of simply reducing the stochastic edge weights to proxy deterministic values). In addition, we showed that for a given level of "possibility threshold" $\epsilon$, each edge density corresponds to a unique worst case value $\bar{x}$. We showed that edge worst case value can be combined into path worst case values, and that the minimum $\bar{x}^{*}$ of such values over a network can be computed using the GDA.

The pair $\bar{x}^{*}$ and $\epsilon$ allow us to compare the worst-case network routing performance. Given a constant benchmark worst-case value $\bar{x}$ for all the networks $\left\{G_{i}\right\}$ under evaluation, we can solve for the $\epsilon_{i}$ value for each network $G_{i}$. The network $G^{*}$ with the lowest value of $\epsilon^{*}$ is the network with the best worst-case routing performance. Alternatively, the $\epsilon$ value can be fixed for all the networks under evaluation. The worst-case values $\bar{x}_{i}$ can then be compared to select the network $G^{*}$ with the best worst-case performance, i.e., the smallest failure rate $\bar{x}_{i}$ among all the networks $G_{i}$.

Future work includes simulating or experimentally verifying the theoretical results presented herein, and generalizing the results further to other types of edge weight densities. Another interesting application would be to use the worst-case performance evaluation method on actual or planned pervasive mission critical sensor network projects.

\section{References}

[1] "ANSS - Advanced National Seismic System," web site address at http://earthquake.usgs.gov/anss/.

[2] "PTWC - Pacific Tsunami Warning Center," web site address at $h t t p: / / w w w . p r h . n o a a . g o v / p r / p t w c /$.

[3] Sobrinho, J.L., "Algebra and algorithms for QoS path computation and hop-by-hop routing in the Internet," IEEE/ACM ToN., vol. 10, pp. 541-550, August 2002.

[4] Stanica, P., "Good Lower and Upper Bounds on Binomial Coefficients," Journal of Inequalities in Pure and Applied Mathematics., vol. 2, no. 3, article 30, 2001.

[5] Cormen, T.H., et al., Introduction to Algorithms, MIT Press, MA and McGraw-Hill, NY, 1990. 